\title{
Una mirada a los clásicos en la formación inicial de maestro desde la Lectura Fácil
}

\author{
Manuel Francisco Romero Olivai \\ Universidad de Cádiz, Cádiz, España \\ Hugo Heredia Ponce ii \\ Universidad de Cádiz, Cádiz, España \\ Paula Rivera Juradoiii(i) \\ Universidad de Cádiz, Cádiz, España
}

\begin{abstract}
Resumen
Presentamos una experiencia desde la que se ha pretendido, por un lado, incluir la Lectura Fácil en la formación inicial de los maestros del grado en Educación Primaria de la Universidad de Cádiz como estrategia para el acercamiento a las obras y autores clásicos para la educación literaria y, por otro lado, abordar esta metodología desde los Proyectos de Trabajo Global. De esta manera, recogemos el desarrollo de la experiencia - desde un punto de vista vivencial - a lo largo de tres momentos: en primer lugar, desde la fase inicial de exploración de los planteamientos de la Lectura Fácil; en segundo lugar, desde los procesos de adaptación del texto clásico; y, por último, desde el diseño de los Proyectos de Trabajo Global a partir del texto adaptado. Los resultados han mostrado un interés creciente por este tipo de propuestas para la compresión y disfrute de los textos clásicos y la necesidad de la formación en esta línea de trabajo entre los futuros docentes.
\end{abstract}

Palabras clave

Lectura Fácil. Clásicos. Proyectos de Trabajo Global. Formación inicial.

\section{Um olhar sobre os clássicos no treinamento inicial de professores desde a Leitura Fácil}

\begin{abstract}
Resumo
Apresentamos uma experiência a partir da qual se pretende, por um lado, incluir a Leitura Fácil na formação inicial de professores do Ensino Fundamental da Universidade de Cádiz como estratégia de abordagem das obras clássicas e autores para uma educação literária e, por outro lado, abordar esta metodologia a partir dos Projetos Globais de Trabalho. Dessa forma, reunimos o desenvolvimento da experiência - do ponto de vista experiencial - em três momentos: primeiro, a partir da fase inicial de exploração das abordagens da Leitura Fácil; segundo, a partir dos processos de adaptação do texto clássico; e, finalmente, a partir do desenho dos Projetos Globais de Trabalho a partir do texto adaptado. Os resultados têm demonstrado um interesse crescente neste
\end{abstract}


tipo de propostas para a compreensão e apreciação dos textos clássicos e a necessidade de formação nesta linha de trabalho entre os futuros professores.

\title{
Palavras-chave
}

Leitura Fácil. Clássicos. Projetos Globais de Trabalho. Treinamento inicial.

\section{A look at the classics in initial teacher training from Easy Reading}

\begin{abstract}
We present an experience from which it has been intended, on the one hand, to include Easy Reading in the initial training of teachers of the degree in Primary Education at the University of Cadiz as a strategy for approaching the classic works and authors for a literary education and, on the other hand, to address this methodology from the Global Work Projects. In this way, we gather the development of the experience - from an experiential point of view - along three moments: firstly, from the initial phase of exploration of the approaches of the Easy Reading; secondly, from the processes of adaptation of the classic text and, finally, from the design of Global Work Projects from the adapted text. The results have shown a growing interest in this type of proposals for the understanding and enjoyment of classical texts and the need for training in this line of work among future teachers.
\end{abstract}

\section{Keywords}

Easy Reading. Classics. Global Work Projects. Initial training.

\section{La Lectura Fácil y los Proyectos de Trabajo Global: punto de encuentro entre dos metodologías}

Esta experiencia surge del encuentro entre estas dos metodologías al considerar que de la unión de la Lectura Fácil (en adelante, LF) - como instrumento fundamental en el ámbito de la diversidad - y de los Proyectos de Trabajo Global (en adelante, PTG) como manera de intervenir que prioriza una perspectiva procedimental en el proceso de enseñanza y aprendizaje - se puede contribuir al acercamiento a los textos y autores clásicos dentro de la formación inicial del docente.

\subsection{La Lectura Fácil}

El movimiento LF se originó en los países nórdicos, concretamente en Suecia. Según explica Mata (2012), impulsora de Lectura Fácil Euskadi:

Educ. Form., Fortaleza, v. 6, n. 1, e3533, jan./abr. 2021

DOI: https://doi.org/10.25053/redufor.v6i1.3533

https://revistas.uece.br/index.php/redufor/index 
La primera iniciativa en la materia nació en 1976 cuando el gobierno sueco contrató a un experto en lingüística para organizar una modernización sistemática del lenguaje en documentos gubernamentales como leyes, decretos y documentos normativos. Más tarde, esta práctica de simplificar el lenguaje se extendió a otros niveles de gobierno, a la academia e incluso a la iniciativa privada.

En España, el método de LF es relativamente nuevo. Desde el año 2002, la Asociación Lectura Fácil trabaja para hacer accesible la lectura, la cultura y la información a todas las personas, con especial atención a aquellas con dificultades lectoras y de comprensión - como pueden ser personas con alguna discapacidad intelectual, ancianos, población inmigrante reciente o alumnos de Educación Primaria, entre otros colectivos (Figura 1), "[...] mediante la aplicación de un conjunto de recomendaciones o pautas lingüísticas y ortotipográficas a las que deben atenerse los materiales creados al amparo de dicho concepto" (ANULA, 2005, p. 4).

Figura 1 - Principales colectivos destinatarios de los materiales de LF

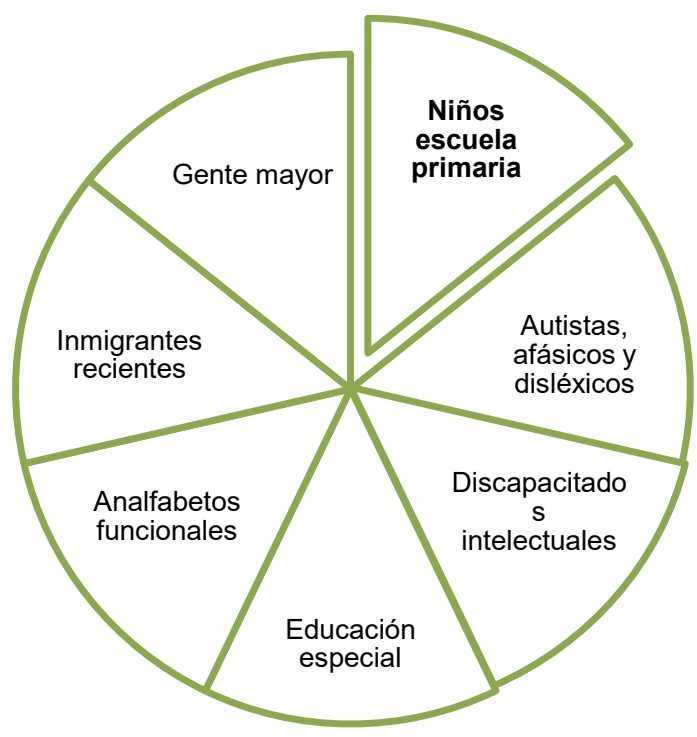

Fuente: Mayol (2003).

Por tanto, parecen dibujarse dos líneas de actuación que justifican que esta metodología se inserte en la formación inicial del futuro maestro: por un lado, los niños de la escuela primaria como uno de los principales colectivos destinatarios de los materiales de LF y, por otro, el acceso a la cultura, la literatura y la información como necesidad y derecho de todas las personas. A menudo, aquellas con dificultades lectoras y de comprensión se ven privadas de este derecho y del placer de leer porque no encuentran libros adecuados a su nivel lector (ANANIAS; SANTOS, 2019). En este

Educ. Form., Fortaleza, v. 6, n. 1, e3533, jan./abr. 2021

DOI: https://doi.org/10.25053/redufor.v6i1.3533

https://revistas.uece.br/index.php/redufor/index 
sentido, en opinión de Salvador (2007), tanto si son dificultades transitorias como permanentes, los textos elaborados según los criterios de LF constituyen un valioso apoyo para ayudarlas a adquirir o a recuperar el placer de leer y a no sentirse excluidas de la sociedad (SALVADOR, 2007).

\subsection{Proyectos de Trabajo Global}

En educación se espera que el docente busque metodologías innovadoras para que el alumnado se integre en el aula y, de esta forma, puedan participar todos indistintamente de sus fortalezas y dificultades para aprender. En este sentido, aparece el aprendizaje basado en proyectos (en adelante, ABP), que, como expresan Esteban y Amador (2017, p. 23):

\footnotetext{
Pretende ofrecer una metodología que rompa con los esquemas tradicionales que rigen actualmente, en la mayoría de los casos, el actual sistema educativo, basándose en un marco teórico que fundamente y sustente estos criterios docentes universitarios. De ahí que el ABP se considere como un método de aprendizaje que favorece el desarrollo de las capacidades, competencias, habilidades y valores del alumnado.
}

Además, el docente va a partir de las ideas previas que tenga y, sobre todo, va a resolver "problemas" de la vida cotidiana desarrollándose la Zona de Desarrollo Próximo del alumno. De ahí que los inicios del ABP estuvieran en el aprendizaje constructivista. En este sentido, Trujillo $(2012$, p. 7$)$ indica que "[...] la enseñanza basada en proyectos 0 tareas integradas supone hoy la mejor garantía didáctica para una contribución eficaz al desarrollo de las competencias básicas y al aprendizaje de los contenidos del currículo". Por lo tanto, desde los primeros niveles de concreción se podrían mejorar aquellos aspectos negativos que se siguen observando en las aulas.

Zabala (2005) distingue cuatro tipos de proyectos: los centros de interés de Decroly, el método de proyectos de Kilpatrick, la investigación del medio del Movimiento Cooperazione Educativa de Italia y los PTG, centrándonos en este último. Este último lo define Domínguez (2003 apud MÉRIDA et al., 2011, p. 66-67) como:

[...] una propuesta de aprendizaje de carácter global, que parte de los intereses del alumnado y se apoya en sus hipótesis de trabajo, articulándose de forma flexible en torno a una temática determinada y 
que, desde la diversidad de los aprendices, se desarrolla en un contexto de interacciones, indagación y actividad permanente.

Como todo proyecto, hay una serie de fases. A continuación, las exponemos, aunque se desarrollarán en el siguiente apartado (ZABALA, 2005):

- Elección del tema;

- Planificación del desarrollo del tema;

- Búsqueda de información;

- Tratamiento de la información;

- Desarrollo de los diferentes apartados del índice;

- Elaboración del dosier de síntesis;

- Evaluación;

- Nuevas perspectivas.

Por lo tanto, la incorporación de este tipo de proyecto en el aula hace que se trabaje de forma interdisciplinar, desarrollándose numerosas materias en una actividad globalizadora (BRANDT; HOBOLD, 2019).

\section{Metodología}

Esta investigación parte del siguiente objetivo general: analizar el conocimiento que tienen los estudiantes en su formación inicial de magisterio sobre la LF. A partir de aquí se desglosan unos objetivos, como son:

a) Estudiar la importancia que tiene para ellos la metodología de ABP en proyectos;

b) Analizar cómo es el proceso de adaptación por parte de los estudiantes.

Además, las miradas y voces implicadas en esta experiencia se desdoblan en dos: por un lado, proyectaremos nuestra visión como mediadores en el contexto de una investigación de corte participativo $\mathrm{y}$, por otro, intentaremos dar la palabra a los estudiantes, los verdaderos protagonistas del proceso.

Para poder desarrollar esta investigación, se siguió una metodología mixta, concretamente un estudio de caso siguiendo las cuatro fases que establecen Rodríguez, Gil y García (1996): reflexiva, trabajo de campo, analítica e informativa.

En cuanto a los participantes, podemos decir que esta experiencia se llevó a cabo en la asignatura de la mención ABP, Competencias Comunicativas en el Currículo 
Integrado del Tercer Curso del Grado en Educación Primaria de la Universidad de Cádiz (España). Esta se desarrolló en el curso 2015-2016, donde participaron un total de 25 alumnos, donde 20 son mujeres y cinco son hombres.

Por otra parte, los instrumentos utilizados para esta investigación fueron el diario, el cuestionario y el grupo de discusión (Tabla 1).

Tabla 1 - La relación de los instrumentos con las fases

\begin{tabular}{|c|c|c|}
\hline Instrumentos & Finalidad & Fase \\
\hline Diario & Para poder apuntar como investigador-docente todas las \\
impresiones. & En todas las fases. \\
\hline Cuestionario & $\begin{array}{c}\text { Con este instrumento queríamos analizar las creencias que } \\
\text { tenían los estudiantes acerca de la LF y el ABP. Y también } \\
\text { para saber la opinión que tenían ellos sobre la experiencia. }\end{array}$ & Reflexiva y analítica. \\
\hline $\begin{array}{c}\text { Grupo de } \\
\text { discusión }\end{array}$ & $\begin{array}{c}\text { Para profundizar en los resultados que se han obtenido en el } \\
\text { cuestionario. }\end{array}$ & Reflexiva y analítica. \\
\hline
\end{tabular}

Fuente: Elaboración propia (2020).

\section{Experiencia}

Esta experiencia se fundamenta en dos objetivos claves: por un lado, incluir la LF en la formación inicial de los maestros como estrategia para el acercamiento a las obras y autores clásicos para la educación literaria y, por otro lado, abordar esta metodología desde los PTG.

Con el fin de completar esa formación del maestro de hoy en día, este trabajo se llevó a cabo por otros tres motivos:

1. Con la LF se trabaja desde la diversidad de alumnos que nos encontramos en nuestras aulas;

2. La LF, a través de sus estrategias, puede acercar los clásicos a los adolescentes, pues nos encontramos que es una lacra en nuestra educación, ya que los alumnos tienen dificultades a la hora de leer por sí solos debido a la complejidad de las estructuras sintácticas, el léxico, de tal forma que no se estaría fomentado el hábito lector;

3. Hay que promover una metodología constructivista donde el alumno sea el protagonista del proceso de enseñanza-aprendizaje. Y, por eso, vemos necesario la incorporación de trabajos globalizados. 
A continuación, a través de las fases, explicaremos cómo fue el proceso de esta experiencia.

\subsection{Fase reflexiva}

En esta fase quisimos, mediante el grupo de discusión y el cuestionario, analizar las creencias previas sobre la LF y el ABP en proyectos.

En cuanto a la LF, el $100 \%$ de los estudiantes no conocían a qué se refería ese concepto y, además, comentaron que era la primera vez que lo habían escuchado. Por otra parte, la mayoría conocía qué era $\mathrm{ABP}$ - esto era obvio, porque, al ser una optativa, la habían seleccionado ellos mismos. A la hora de preguntarles qué les sugiere el ABP, estos fueron los resultados: "Trabajar a través de las competencias para que el alumno adquiera de una mejor forma de los contenidos" (ALUM_14)1; "Me sugiere trabajar entre varias asignaturas sin tener compartimiento estancos" (ALUM_11); "Colaboración entre los estudiantes y los docentes para llevar a cabo una clase" (ALUM_02).

\subsection{Fase trabajo de campo}

Una vez analizada la situación de partida, comenzamos a establecer nuestra propuesta, que queda dividida en las siguientes fases:

Fase 1. Exploración de la metodología $L F$

El desconocimiento de los estudiantes universitarios sobre la metodología de la LF nos llevó a dedicar la primera sesión de la experiencia a explorar, por un lado, las Directrices Europeas de la International League of Societies for Persons with Mental Handicaps (ILSMH) y, por otro, los orígenes de la Asociación Lectura Fácil, sus planteamientos y su justificación en la formación inicial del maestro.

Ambas instituciones sirvieron para centrar el punto de partida de la propuesta metodológica e iniciar nuevas lecturas para la formación de los estudiantes desde los

1 Para guardar el anonimato de los estudiantes, se ha codificado con ALUM y un número (ALUM_nº).

Educ. Form., Fortaleza, v. 6, n. 1, e3533, jan./abr. 2021

DOI: https://doi.org/10.25053/redufor.v6i1.3533

https://revistas.uece.br/index.php/redufor/index 
presupuestos de la Declaración Universal de los Derechos Humanos: "Toda persona tiene derecho a tomar parte libremente en la vida cultural de la comunidad, a gozar de las artes y a participar en el progreso científico y en los beneficios que de él resulten" (ONU, 1948).

Fase 2. Adaptación del capítulo de los molinos de viento del Quijote a LF

Tras la presentación de los principios que justifican la LF, el paso siguiente se centró en adaptar un fragmento del Quijote para que fuese fácil de leer y fácil de entender. Antes de abordar esta tarea, los estudiantes necesitaron previamente conocer las pautas que esta metodología exige, así como realizar diversos ensayos antes de lograr la adaptación definitiva, que sería la que les sirviera de base sobre la que fundamentar el PTG.

Fase 2a) Pautas iniciales

Esta fase se orientó hacia el conocimiento de las pautas que exige la LF antes de iniciar el proceso de adaptación del texto; de tal manera, que la sesión supuso reflexionar sobre:

1. El contenido: ¿qué queremos comunicar?, ¿con qué finalidad?

2. El destinatario: ¿cuáles son las características del público al que me dirijo?

3. El soporte: ¿en qué soporte voy a presentar los contenidos?

4. Los criterios de adaptabilidad del texto escogido.

La web Universo Cervantes sirvió de referencia y análisis a estas preguntas de partida, pues en ella se encuentran algunos ejemplos de adaptaciones que ayudaron a los estudiantes a la realización del primer ensayo.

Fase 2b) Ensayos

La fase dedicada a realizar los ensayos tomó como referencia los planteamientos de Anula (2005) sobre los distintos procesos por los que pasan los textos que se adaptan a LF (Figura 2):

Educ. Form., Fortaleza, v. 6, n. 1, e3533, jan./abr. 2021

DOI: https://doi.org/10.25053/redufor.v6i1.3533

https://revistas.uece.br/index.php/redufor/index 
1. Planificación de la adaptación y elaboración de la guía de contenidos;

2. Adaptación lingüística del texto (procesos de simplificación léxica, morfosintáctica, textual e inferencial o pragmática).

3. Adaptación icónica y ortotipográfica.

4. Revisión de la adaptación.

Figura 2 - Proceso de adecuación lingüística, visual y ortotipográfica en LF

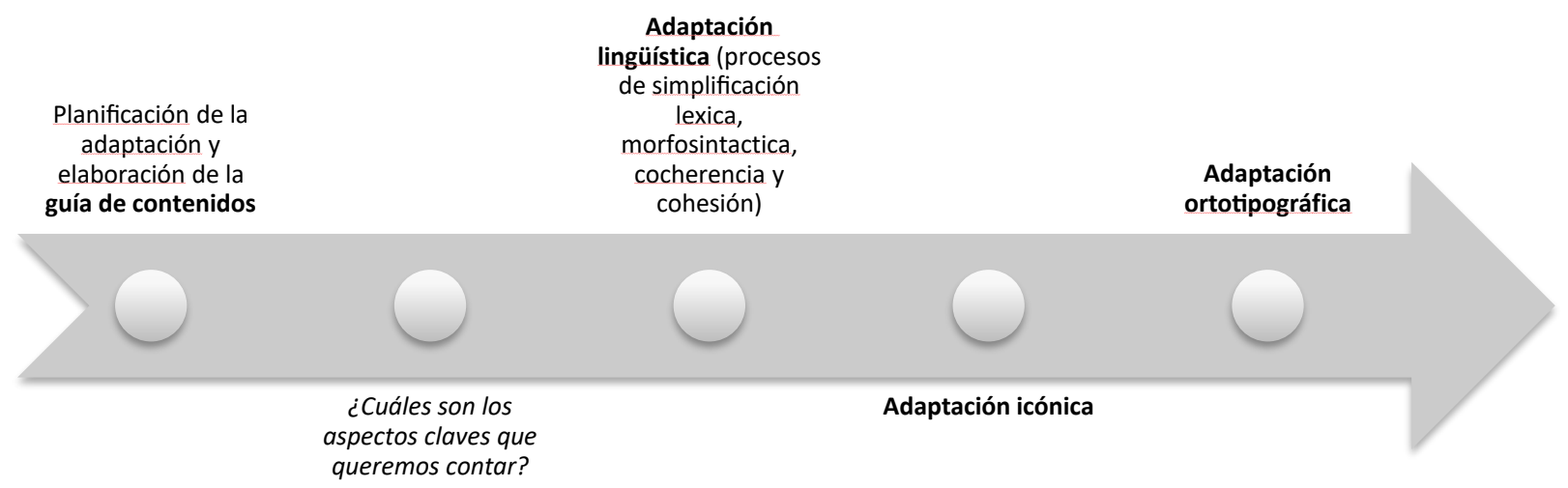

Fuente: Elaboración propia de las fases propuestas por Anula (2007).

Fase 2c) Adaptación definitiva

Para el proceso de validación de la adaptación, el texto se revisó atendiendo a los parámetros que establece Anula (2005):

1. La adaptación debe ser fiel a la intención y al sentido del texto;

2. La adaptación debe recoger la acción principal del texto;

3. La adaptación no debe resultar infantil;

4. La adaptación lingüística debe responder a criterios de simplicidad.

No obstante, deben ser también aquellos lectores destinatarios de esta quienes deban aprobar el texto adaptado. De ahí, el interés de incluir esta adaptación como punto de partida de un proyecto globalizado para lectores de Educación Primaria y esta debe realizarse, siguiendo a Salvador (2007), teniendo en cuenta la reflexión metalingüística del docente que actúa como mediador en el aula: 


\section{En cuanto al léxico:}

- ¿Se han evitado los conceptos abstractos?

- ¿Se han empleado palabras de uso habitual?

- ¿Se ha procurado ser sistemático al utilizar las palabras (a pesar del hecho de que la repetición haya afectado al estilo de redacción)?

- ¿Se han evitado palabras de otro idioma?

- ¿Se ha evitado el uso de jergas, abreviaturas o iniciales?

- ¿Se repiten sustantivos para nombrar a los personajes durante toda la obra?

- ¿Se han buscado sinónimos para aquellas palabras que puedan haber quedado en desuso?

- ¿Se han buscado sinónimos para facilitar la comprensión?

\section{En cuanto a la sintaxis:}

- ¿Se recurre a estructuras paralelísticas respetando el orden de sujeto + verbo + complementos?

- ¿Se evitan las estructuras complejas?

- ¿Se emplean, en su mayoría, oraciones cortas?

- ¿Se incluye una sola idea principal en cada oración?

- ¿Se emplea un lenguaje positivo evitando las negaciones, ya que suelen crear confusión?

- ¿Se ha empleado la voz activa frente a la pasiva?

- ¿Se evita el empleo del subjuntivo?

\section{En cuanto a la cohesión:}

- ¿La coesión asegura la comprensión global del significado del texto?

- ¿Se separan las partes narrativas de las dialogadas mediante líneas en blanco?

- ¿Cada párrafo conforma una unidad semántica o textual?

- ¿Cada párrafo está formado por un conjunto de oraciones, separadas entre sí mediante el punto y aparte?

- ¿Se conservan los conectores discursivos presentes en la obra original, siempre y cuando no resulten arcaicos ni supongan una dificultad añadida para la comprensión total del texto? 
Fase 3. Diseño de un Proyecto de Trabajo Global

Para el diseño del proyecto, los estudiantes han seguido las fases que propone Zabala (2005, p. 171-172) para la realización de un PTG:

1. Elección del tema. El tema del proyecto gira en torno a El Quijote y Miguel de Cervantes, debido a la conmemoración, durante el curso 2015/2016, del cuarto aniversario de la muerte del autor. Este acontecimiento va a suponer el eje vertebrador de un proyecto que va a recoger no solo el conocimiento del autor y la obra, sino también otros aspectos, como el contexto histórico o la relación con otros autores universales. Todo ello permitirá diseñar un proyecto integrador y globalizado partiendo del texto adaptado a LF.

2. Planificación del desarrollo del tema. Después de la elección del tema, cada grupo realizó una propuesta de índice de los diferentes apartados del proyecto. Asimismo, establecieron cómo distribuir el tiempo y las tareas a realizar en distintas sesiones para la búsqueda de la información que tiene que dar respuesta a los apartados del índice. De este modo, los estudiantes han planificado un proyecto para ser desarrollado a lo largo de 10 sesiones y destinado al tercer ciclo de Educación Primaria Obligatoria, concretamente para $6^{\circ}$ curso. Para la planificación de cada sesión, han debido cumplimentar una ficha como la que se muestra en la Tabla 2, indicando título de la sesión, fecha, objetivos, contenidos, materiales, evaluación, rutinas de trabajo y cualquier otra observación.

Tabla 2 - Ficha de planificación de sesiones

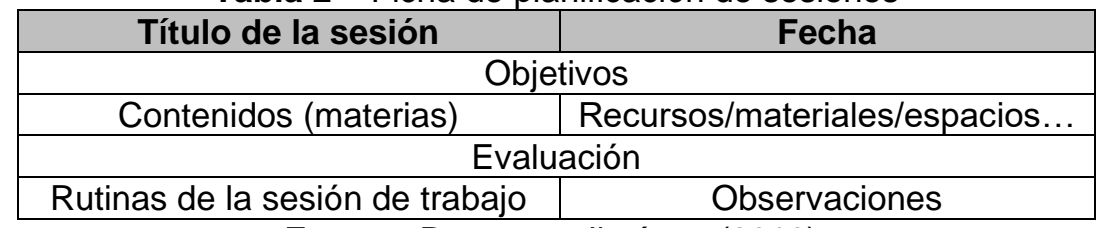

Fuente: Romero y Jiménez (2016).

Igualmente, una segunda ficha - Tabla 3 - señalando aquellas competencias claves de la sesión y de qué manera se está contribuyendo a su adquisición y desarrollo. 
Tabla 3 - Ficha para la adquisición y desarrollo de las competencias clave

\begin{tabular}{|c|c|}
\hline Competencias clave & $\begin{array}{c}\text { Contribución a la adquisición y } \\
\text { desarrollo }\end{array}$ \\
\hline Comunicación lingüística & \\
Competencia matemática y & \\
y tecnología & \\
Competencia digital & \\
Aprender a aprender & \\
Competencias sociales y cívicas & \\
Sentido de la iniciativa y espíritu & \\
emprendedor & \\
Conciencia y expresiones culturales & \\
\hline
\end{tabular}

Fuente: Romero (2016) adaptadas a las competencias claves.

3. Búsqueda de información. Una vez configurado el índice como guion de trabajo, los estudiantes pasaron a seleccionar aquellos enlaces más apropiados y accesibles para que los alumnos de 6ำ de Educación Primaria buscaran la información que diese respuesta a aquellos apartados del índice. En la Tabla 4 puede verse una propuesta de guion de trabajo de uno de los grupos.

Tabla 4 - Apartados del guion de trabajo

\begin{tabular}{|c|c|}
\hline 1 & Introducción \\
\hline 2 & Cervantes y El Quijote \\
\hline 2.1 & Cervantes: \\
& $\begin{array}{c}\text { - Contexto histórico de la época } \\
\text { - Vida } \\
\text { - Obras }\end{array}$ \\
\hline 2.2 & $\begin{array}{c}\text { El Quijote } \\
\text { - Argumento } \\
\text { - Personajes }\end{array}$ \\
\hline 2.3 & Conexión entre Cervantes y Shakespeare \\
\hline 2.4 & Las huellas y el legado de El Quijote \\
\hline
\end{tabular}

De este modo, los estudiantes han intentado seleccionar enlaces de distinta complejidad lingüística para lograr la accesibilidad a la información de todos los alumnos de este ciclo. Asimismo, bajo este criterio, han facilitado espacios virtuales en distintos formatos: presentaciones, vídeos, textos y fotografías.

4. Tratamiento de la información. Según Zabala (2007, p. 158), esta es una de las fases más significativas del proyecto, ya que el alumno tiene que:

Poder seleccionar y reconocer lo que es esencial de lo que es anecdótico; distinguir entre hipótesis, teorías, opiniones y puntos de vista; adquirir las habilidades para trabajar con medios y recursos diferentes; clasificar y ordenar la 
información; llegar a conclusiones y establecer procesos de descontextualización para poder generalizar $y$, finalmente, plantear nuevas preguntas.

Por ello, para abordar esta fase, los estudiantes han diseñado para los destinatarios del proyecto unas rutinas de trabajo bien definidas que se concretan en:

a) Apertura de la sesión. El docente abre la sesión pidiendo a cada grupo de alumnos que exponga al resto la información recopilada en sesiones anteriores;

b) Tratamiento de la información. Con el fin de seleccionar la información relevante y adquirir las habilidades más significativas de esta fase, los alumnos han de elaborar resúmenes y otras tareas de síntesis, así como reelaboración de enunciados complejos y búsqueda de palabras desconocidas;

c) Cierre de la sesión. Al finalizar la sesión, el docente pide a cada grupo de alumnos una lectura comprensiva de todo lo elaborado para verificar la rutina de trabajo anterior y corregir errores.

5. Desarrollo de los diferentes apartados del índice. A partir del tratamiento de la información, van a elaborarse los contenidos de los diferentes apartados que configuran el índice con vistas al diseño del dosier de síntesis.

6. Elaboración del dosier de síntesis. El dosier de síntesis supone la concreción de todo el trabajo anterior, es decir, el producto del proyecto. En esta experiencia, el dosier de síntesis ha tomado forma de dosier, libro artesanal o home-made book, realizados por los estudiantes, con una perspectiva integradora e interdisciplinar, como podemos observar en la Imagen 1.

\section{Imagen 1 - Ejemplo de libro artesanal: laap-book de El Quijote}

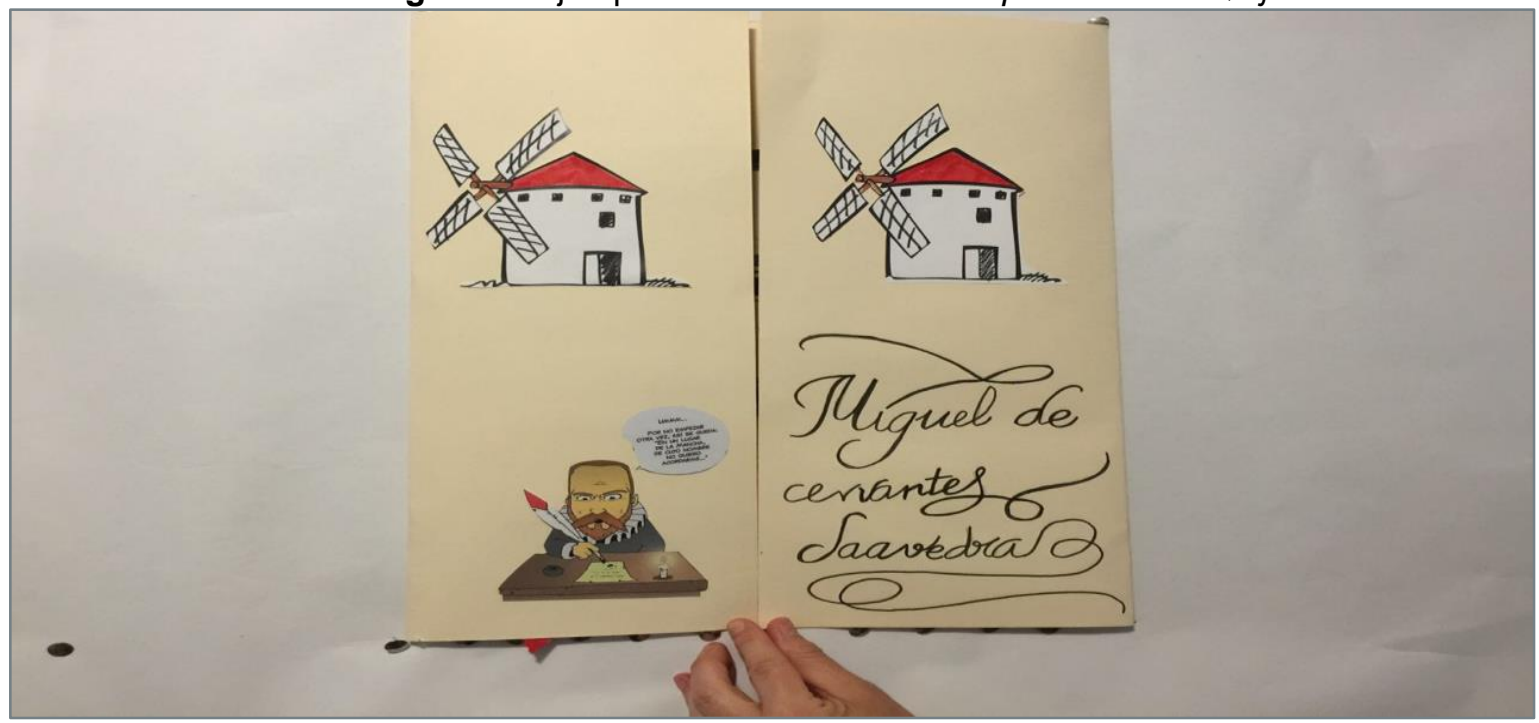

Educ. Form., Fortaleza, v. 6, n. 1, e3533, jan./abr. 2021

DOI: https://doi.org/10.25053/redufor.v6i1.3533

https://revistas.uece.br/index.php/redufor/index 
En definitiva, este producto final va a servir de eje central para el desempeño de las competencias clave recogidas en la Ley Orgánica para la Mejora de la Calidad Educativa (LOMCE) y su carácter integrador va a contribuir al desarrollo de contenidos y objetivos de varias asignaturas.

7. Evaluación. El proceso final se evalúa, según Zabala (2007, p. 158-159), en dos niveles:

\footnotetext{
Uno de índole interna, que realiza cada chico y chica y en el cual recapitula sobre lo que ha hecho y lo que ha aprendido; y otro de índole externa, en el cual, con ayuda del profesor, los alumnos tienen que profundizar en el proceso de descontextualización, aplicando a situaciones diferentes la información trabajada y las conclusiones obtenidas, estableciendo relaciones y comparaciones que permitan la generalización y la conceptualización.
}

De este modo, aquellos instrumentos que los estudiantes han propuesto para la evaluación interna han sido dos: por una parte, el informe de síntesis, de carácter grupal, donde los alumnos han de reflejar sus logros y dificultades durante todo el proceso, el rol que ha adoptado cada componente del grupo y todos aquellos aspectos que han quedado abiertos y les ha suscitado interés por indagar. Y, por otra, un documento de autoevaluación, de carácter individual, donde ha de quedar reflejada la postura del alumno frente al trabajo y al proyecto en sí realizado.

Para la evaluación externa, los estudiantes han propuesto una sesión dedicada a exposiciones orales donde cada grupo presentará su dosier de síntesis final, el homemade book, destacando aquellos obstáculos, temores y motivaciones que se han dado durante el desarrollo de la experiencia.

\subsection{Fase analítica}

Una vez terminada la experiencia, se pretendió conocer cuáles eran sus impresiones, es decir, valorar la experiencia.

En un primer momento les preguntamos qué les había parecido la experiencia, y el $100 \%$ de los estudiantes indicaron que había sido muy buena. Además, quisimos saber qué opinión tenían ellos sobre cómo la LF puede contribuir al gusto por la lectura: "En la medida en la que el alumno es capaz de codificar y comprender el texto de inmediato, sin tener dificultades a la hora de leer" (ALUM_5); "Ayuda a motivar al 
lector a seguir leyendo" (ALUM_17); "Contribuye favorablemente, puesto que el niño así puede entenderlo todo y así interesarse por lo que está leyendo" (ALUM_2).

Por otra parte, el $100 \%$ de los estudiantes comentaron que la LF ayuda al acercamiento de los clásicos entre los más pequeños, por los siguientes motivos que algunos indicaron: "Es una forma de hacer ver a nuestros alumnos que, a partir de un texto clásico, se puede adaptar de manera que ellos lo entiendan mejor y, de esta forma, se sientan mas atraídos por la obra" (ALUM_9); "Porque lo comprenden mejor" (ALUM_1); "Porque normalmente los clásicos utilizan un lenguaje poco cercano al alumnado y así puede leerlo todo comprendiéndolo" (ALUM_20).

\subsection{Fase informativa}

Tras terminar la experiencia y obtener los resultados, comentamos con la clase para poder replicarla en los demás cursos académicos y así fue: en los cursos sucesivos, se ha querido adaptar a LF un clásico y trabajar a través del ABP.

\section{Conclusiones}

La valoración y conclusiones de la experiencia se plantean desde la línea de mejora del potencial creativo del docente y la mejora de la formación docente (KONDRASHOVA et al., 2020), de ahí la importancia de contrastar con los propios testimonios de los implicados:

Desde la formación inicial y las vivencias de los futuros maestros:

a) La valoración de la experiencia fue altamente positiva y les permitió afrontar situaciones específicas de un aula real: fomento de la lectura, búsqueda de estrategias de acercamiento a los textos clásicos, atención a la diversidad, dificultades de aprendizaje, entre otras;

b) El descubrimiento de la metodología en LF y su inclusión en los PTG les ha ofrecido una nueva alternativa desde la que contribuir a la comprensión y goce de lectura de textos clásicos entre la población infantil:

En la medida en la que el alumno es capaz de codificar y comprender el texto de inmediato, sin tener dificultades a la hora de leer. (ALUM_5).

Educ. Form., Fortaleza, v. 6, n. 1, e3533, jan./abr. 2021

DOI: https://doi.org/10.25053/redufor.v6i1.3533

https://revistas.uece.br/index.php/redufor/index 
Ayuda a motivar al lector a seguir leyendo. (ALUM_17).

Contribuye favorablemente, puesto que el niño así puede entenderlo todo y así interesarse por lo que está leyendo. (ALUM_2).

Es una forma de hacer ver a nuestros alumnos que, a partir de un texto clásico, se puede adaptar de manera que ellos lo entiendan mejor y, de esta forma, se sientan más atraídos por la obra. (ALUM_9).

Porque normalmente los clásicos utilizan un lenguaje poco cercano al alumnado, y así puede leerlo todo comprendiéndolo. (ALUM_1).

Desde nuestra visión como mediadores de la experiencia:

a) Consideramos que hemos dado respuesta a aquellas competencias y resultados de aprendizajes que aparecen en el diseño de diversas asignaturas del itinerario curricular del Grado en Educación Primaria, tales como diseñar programas de intervención para el tratamiento de las diferencias y dificultades de aprendizaje;

b) La grata acogida de la experiencia nos ha sugerido la continuidad de esta línea de trabajo con la adaptación a LF de otros textos clásicos de la literatura española y universal y su proyección desde las posibilidades de los PTG.

\section{Referencias}

ANANIAS, M.; SANTOS, L. R. Quando o homem sabe ler, escrever e contar, pode, por sua própria individualidade, desenvolver-se e esclarecer-se": a escolarização de crianças pobres na província da Parahyba do Norte (1855-1866). Educação \& Formação, Fortaleza, v. 4, n. 1, p. 66-80, 2019. Disponible en:

https://revistas.uece.br/index.php/redufor/article/view/453/1082. Acceso el: 29 jul. 2020.

ANULA, A. (Dir.). Introducción a Don Quijote de la Mancha de Fácil Lectura. Madrid: Fundación Universidad Autónoma de Madrid, 2005.

BRANDT, A.; HOBOLD, M. S. Prática como componente curricular na disciplina Pesquisa e Processos Educativos do curso de Pedagogia: um diferencial na relação entre pesquisa, teoria e prática. Educação \& Formação, Fortaleza, v. 4, n. 2, p. 142-160, 2019. Disponible en: https://revistas.uece.br/index.php/redufor/article/view/319/1146. Acceso el: 29 jul. 2020.

ESTEBAN, M.; AMADOR, L. V. Modificación de conductas ambientales desde el aprendizaje basado en proyectos. MoleOla: Revista de Ciencias de la Universidad Pablo de Olavide, Sevilla, n. 25, p. 23-25, 2017.

ILSMH. El Camino Más Fácil. 1998a. Disponible en:

http://www.lecturafacil.net/media/resources/ilsmhcastellà.pdf. Acceso el: 12 abr. 2020.

ILSMH. El Camino Más Fácil. Directrices europeas para generar información de Fácil Lectura destinada a personas con retraso mental. 1998b. Disponible en: 
http://portal.uc3m.es/portal/page/portal/orientacion_personal_participacion/pied1/lectura facil/documentos/manualdelecturafacil.pdf. Acceso el: 4 abr. 2020.

KONDRASHOVA, L. V. et al. Desenvolvimento do potencial criativo de futuros professores: estratégia para melhorar a qualidade do ensino superior pedagógico. Educación \& Formación, Fortaleza, v. 5, n. 3, p. 1-15, 2020. Disponible en: https://revistas.uece.br/index.php/redufor/article/view/3292/2917 Acceso el: 3 jul. 2020.

MATA, B. Claro, útil, comprensible. 2012. Disponible en: https://lecturafacileuskadi.net/blog/claro-util-lenguaje-llano/. Acceso el: 10 mayo 2020.

MAYOL, M. C. Concepto de "Lectura Fácil y tipología de usuarios". Educación y Biblioteca, La Rioja, n. 136, p. 76-80, 2003.

MÉRIDA, R. et al. Aprender investigando en la escuela y en la Universidad. Una experiencia de investigación-acción a partir del Trabajo por Proyectos. Investigación en la Escuela, Sevilla, n. 73, p. 65-76, 2011.

ONU. Declaración Universal de Derechos Humanos (10 diciembre 1948, 217 A (III)). Disponible en: https://www.refworld.org.es/docid/47a080e32.html. Acceso en: 14 mayo 2020.

RODRÍGUEZ, G.; GIL, J.; GARCÍA, E. Metodología de la investigación cualitativa. Málaga: Aljibe, 1996.

ROMERO, M. F.; JIMÉNEZ, R. La lectura intensiva como estrategia para el desarrollo de las habilidades comunicativas entre los estudiantes de secundario ELE. In: WILK-RACIĘSKA, J.; SZYNDLER, A.; TATOJ, C. (Ed.). Relecturas y nuevos horizontes en los estudios hispánicos: lingüística y didáctica. Wydawnictwo: Uniwersytetu Śląskiego, 2016. p. 333-356.

SALVADOR, E. Estimular la lectura: las posibilidades del material de lectura fácil. Aula de Innovación Educativa, Barcelona, n. 167, p. 27-29, 2007.

TRUJILLO, F. Enseñanza basada en proyectos: una propuesta eficaz para el aprendizaje y el desarrollo de las competencias básicas. Revista Eufonía: Didáctica de la Educación Musical, Barcelona, n. 55, p. 7-15, 2012.

ZABALA, A. Enfoque globalizador y pensamiento complejo: una respuesta para la comprensión e intervención en la realidad. Barcelona: Graó, 2005.

ZABALA, A. La práctica educativa: cómo enseñar. Barcelona: Graó, 2007.

\footnotetext{
Manuel Francisco Romero Oliva, Universidad de Cádiz, Departamento de Didáctica de la Lengua y la Literatura

i D https://orcid.org/0000-0002-6854-0682

Doctor y profesor del Departamento de Didáctica de la Lengua y la Literatura de la Universidad de Cádiz. Sus líneas de investigación se centran en la educación lingüística y literaria en una perspectiva interdisciplinar, donde confluyen diferentes áreas de conocimiento. Participa en varias publicaciones y, en la actualidad, su trabajo se centra en la educación literaria no impartida en las escuelas medias y secundarias, así como en conferencias y cursos en las escuelas.

Contribución de autoría: Fundamentación, diseño metodológico y conclusiones.

E-mail: manuelfrancisco.romero@uca.es
}

Educ. Form., Fortaleza, v. 6, n. 1, e3533, jan./abr. 2021

DOI: https://doi.org/10.25053/redufor.v6i1.3533

https://revistas.uece.br/index.php/redufor/index ISSN: 2448-3583 
Hugo Heredia Ponce, Universidad de Cádiz, Departamento de Didáctica de la Lengua y la Literatura ii(D)http://orcid.org/0000-0003-3657-1369

Doctor y profesor del Departamento de Didáctica de la Lengua y la Literatura en la Universidad de Cádiz. Las investigaciones se centran en la lectura entre los adolescentes y el uso de las nuevas tecnologías.

Contribución de autoría: Diseño y experimentación en aula, recogida de datos y conclusiones.

E-mail: hugo.heredia@uca.es

Paula Rivera Jurado, Universidad de Cádiz, Departamento de Didáctica de la Lengua y la Literatura iii Dhttps://orcid.org/0000-0002-4191-1367

Doctora y profesora del Departamento de Didáctica de la Lengua y la Literatura de la Universidad de Cádiz. Sus líneas de investigación se centran en los procesos de docencia y recepción literaria. Actualmente sus estudios abordan la formación lectora y literaria desde un enfoque intertextual, intercultural e interdisciplinario.

Contribución de autoría: Diseño y experimentación en aula, recogida de datos y conclusiones.

E-mail: paula.rivera@uca.es

Editora responsable: Lia Machado Fiuza Fialho Pareceristas ad hoc: Christian Alejandro Arenas Delgado y Celia Sanz Pérez

\section{Cómo citar este artículo (ABNT):}

ROMERO OLIVA, Manuel Francisco; HEREDIA PONCE, Hugo; RIVERA JURADO, Paula. Una mirada a los clásicos en la formación inicial de maestro desde la Lectura Fácil. Educ. Form., Fortaleza, v. 6, n. 1, e3533, 2020. Disponible en:

https://revistas.uece.br/index.php/redufor/article/view/3533

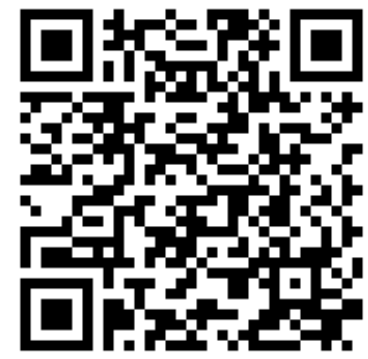

Recibido el 13 de julio de 2020.

Aceptado el 4 de agosto de 2020.

Publicado el 25 de septiembre de 2020.

Educ. Form., Fortaleza, v. 6, n. 1, e3533, jan./abr. 2021

DOI: https://doi.org/10.25053/redufor.v6i1.3533

https://revistas.uece.br/index.php/redufor/index 